\title{
Micropropagation and Ex Vitro Rooting of Wolfberry
}

\author{
Cristian Silvestri' ${ }^{1}$, Gianmarco Sabbatini, Federico Marangelli, \\ Eddo Rugini, and Valerio Cristofori \\ Department of Agriculture and Forest Sciences (DAFNE), University of \\ Tuscia, Via San Camillo De Lellis, s.n.c. - 01100, Viterbo, Italy
}

Additional index words. Lycium barbarum, axillary shoot proliferation, in vitro and ex vitro rooting, acclimatization, growth regulators

\begin{abstract}
An accurate protocol for the in vitro propagation of a commercial wolfberry (Lycium barbarum L.) cv. Nixia 1 has been developed through axillary shoot proliferation. Driver and Kuniyuki Walnut (DKW) medium supplemented with 6-benzylaminopurine (BAP; $0.5 \mathrm{mg} / \mathrm{L}$ ) and sucrose $3 \% \mathrm{w} / \mathrm{v}$ gave the best results compared with other basal media tested, with significantly improved production of multiple shoots direct from nodal segment explants, resulting in an average of 6.73 shoots/explant with an average of 7.45 nodes/shoot that would potentially form new explants. Rooting of shoot explants was carried out both in vitro and ex vitro with 0.5 and $1 \mathrm{mg} / \mathrm{L}$ of indole-3-butyric acid (IBA), with or without adding putrescine $(160 \mathrm{mg} / \mathrm{L})$. In all cases, rooting efficiency resulted very high, and putrescine was effective only when combined with a low concentration of auxin. Plantlets were hardened off in jiffy pots under greenhouse conditions, with a survival rate of more than $90 \%$. Ex vitro rooting, performed by dipping in an aqueous solution of IBA $100 \mathrm{mg} / \mathrm{L}$, is the preferred technique not only because rooting and acclimatization are very high but also reducing micropropagation to one phase is more economical.
\end{abstract}

Wolfberry or goji berry (Lycium barbarum L.) is a member of the Solanaceae family, rich in bioactive polysaccharides (Seeram, 2008). It belongs to the genus Lycium, in which there are more than 70 species, with three main species distributed in the East and South-East Asia, South Africa, and North America (Fukuda et al., 2001).

The species L. barbarum and L. chinense are very similar and have a long tradition as food and medicinal plants in China and other Asian countries. There are also some varieties and related species such as Lycium barbarum var. aurantiocarpum, L. chinense var. potaninii, L. ruthenicum, and L. truncatum, often commercialized as low-cost adulterants (Zhang et al., 2001). For these reasons, the adulteration of commercial products cannot be excluded, and morphological, histological, and molecular differentiations are necessary (Potterat 2010; Sze et al., 2008; Zhang et al., 2001).

Large-scale propagation of woody plant species through in vitro culture techniques represents a rapid way to produce uniform materials in a short time to meet agriculture needs. The application of micropropagation for commercial-scale plant production has been well demonstrated in several plant species that contain potentially useful sec-

Received for publication 24 July 2018. Accepted for publication 20 Aug. 2018.

The research was partially supported by MIUR (MInistry for education, University and Research), Law 232/2016, "Department of excellence" and by funding of Tuscia University DAFNE.RSA16SIL (Ricerca Scientifica Ateneo Silvestri). We thank Antonella Minandri for her work and well-qualified assistance in in vitro culture.

${ }^{1}$ Corresponding author. E-mail: silvestri.c@unitus.it. ondary metabolites (Frabetti et al., 2009). Many of these species have shown excellent in vitro performance in terms of the number of explants produced in a short period of time and offer the opportunity to enhance the amount of these scarce compounds for extractive manipulations (Nalawade et al., 2003; Okemo et al., 2003; Osman et al., 2012, 2013a, 2013b; Osuna et al., 2006; Parveen et al., 2005).

Wolfberry plants often are reproduced through seeds and show all the problems related to sexual propagation, i.e., low germination, absence of clonal fidelity, and nonuniform agronomical performance. Despite the increasing interests in goji cultivation, there are few papers in the scientific literature regarding the micropropagation of wolfberry (Fira et al., 2016), and almost all described micropropagation protocols start from seedlings. Our previous experiments suggested that the major problems hampering the development of in vitro propagation protocols are the deleterious postexposure effect of wolfberry varieties on Murashige and Skoog (MS) medium and the nonuniformity of the explants used for rooting induction.

On the contrary, numerous papers have described regeneration by adventitious shoot organogenesis and somatic embryogenesis, and Agrobacterium-mediated transformation and transgenic plantlets have been obtained by using adventitious organogenesis from different explants (Cui et al., 1998, 1999; Hu et al., 2001, 2002, 2006).

For many fruit species that are not easy to root and acclimatize, the rooting phase has been successfully performed ex vitro and the plants then survived when planted in the field
(Benmahioul et al., 2012; Saiju, 2005). The present work has been conducted to develop an efficient and reliable protocol for the micropropagation of wolfberry (Lycium barbarum L.), focusing on the effects of mineral salts, growth regulators, and in vitro and ex vitro rooting. The simplicity of the present protocol aims not only to promote the propagation of elite cultivar but also the in vitro conservation of interesting genotypes, usable for conventional and unconventional breeding and biotechnologies.

\section{Material and Methods}

Plant material. For in vitro establishment, we started with 1.5 - to $2.0-\mathrm{cm}$-long nodal explants of $L$. barbarum cv. Nixia 1, collected from greenhouse-potted plants at the end of August. All leaves were removed and nodal segments were immersed for $1 \mathrm{~h}$ in aqueous solution containing ascorbic acid $250 \mathrm{mg} / \mathrm{L}$, citric acid $250 \mathrm{mg} / \mathrm{L}$, gibberellic acid $\left(\mathrm{GA}_{3}\right) 5 \mathrm{mg} / \mathrm{L}$ and $\mathrm{PPM}^{\circledR} 0.1 \%$. Then, the explants were surface sterilized in $20 \%$ commercial bleach plus a few drops of Tween 20 for $30 \mathrm{~min}$ and rinsed twice with sterile distilled water. Culture establishments have been performed in $100 \times 20$-mm glass tubes containing $5 \mathrm{~mL}$ of medium comprising halfstrength MS medium (Murashige and Skoog, 1962) supplemented with 6-benzylaminopurine (BAP; $0.5 \mathrm{mg} / \mathrm{L}), \mathrm{GA}_{3}(2 \mathrm{mg} / \mathrm{L})$, and sucrose $3 \%(\mathrm{w} / \mathrm{v})$. The $\mathrm{pH}$ of the medium was adjusted to 5.8 with potassium hydroxide $(\mathrm{KOH}) \quad 1 \mathrm{M}$ before adding $0.58 \%(\mathrm{w} / \mathrm{v})$ of plant agar (Duchefa Biochemie, B.V., Haarlem, The Netherlands). All media used in the experiments were autoclaved $20 \mathrm{~min}$ at $121{ }^{\circ} \mathrm{C}, 1 \mathrm{~atm}$, and all growth regulator were filter-sterilized $(0.22 \mu \mathrm{m})$ and added after the media sterilization. The explants were incubated in a growth chamber at $24 \pm 1{ }^{\circ} \mathrm{C}$ under a 16 -h photoperiod with $40 \mu \mathrm{mol} \cdot \mathrm{m}^{-2} \cdot \mathrm{s}^{-1}$ light intensity provided by cool-white fluorescent lamps. To increase the stock material for the in vitro experiments, the explants were cut and subcultured at 21-d intervals for 3 months.

Growth regulators and culture conditions. To test the plant growth regulators, MS medium supplemented with sucrose $3 \% \mathrm{w} / \mathrm{v}$ was used. The $\mathrm{pH}$ of the medium was adjusted to 5.8 with $\mathrm{KOH} 1 \mathrm{M}$ before adding $0.58 \%(\mathrm{w} / \mathrm{v})$ of plant agar (Duchefa Biochemie, B.V.). Growth regulators were added filter-sterilized. The following growth regulators were used varying different concentrations: BAP $(0,0.5,1,2 \mathrm{mg} / \mathrm{L}), N 6-(2-$ isopentenyl)adenine (2-iP; $0,0.5,1,2 \mathrm{mg} / \mathrm{L})$, Zeatin (ZR; 0, 0.5, 1, $2 \mathrm{mg} / \mathrm{L})$, and control (hormone-free medium).

Eight $500-\mathrm{mL}$ jars containing $50 \mathrm{~mL}$ of culture media were used for each treatment. Twelve explants were subcultured per jar and incubated at the same light and temperature conditions as described for the establishment of the plant material. The number of nodes/ shoot and number of shoots per explant were recorded at the end of the 4-week culture period. The experiment was repeated twice. 
The total number of nodes obtainable from a single explant at the end of each subculture cycle represented possible explants to start a new multiplication cycle. This was calculated using the multiplication factor (MF) by multiplying the average number of shoots per explant by the average of nodes per shoot (Frabetti et al., 2009). Furthermore, the presence or absence of callus production was recorded.

Mineral basal salts and culture conditions. MS (Murashige and Skoog, 1962), Quoirin and Lepoivre (QL; Quoirin and Lepoivre, 1977), DKW (Driver and Kuniyuki, 1984), and Woody Plant Medium (WPM; Lloyd and McCown, 1980) media were used in the second experiment. All media were supplemented with BAP $0.5 \mathrm{mg} / \mathrm{L}$, on the basis of the previous results, sucrose $3 \% \mathrm{w} / \mathrm{v}$, and $\mathrm{pH}$ was adjusted to 5.8 with $\mathrm{KOH} 1 \mathrm{~m}$ before $0.58 \%$ (w/v) of plant agar (Duchefa Biochemie, B.V.) was added.

Eight $500-\mathrm{mL}$ jars containing $50 \mathrm{~mL}$ of culture media were used for each treatment. Twelve explants were subcultured per jar and incubated at the same light and temperature conditions as described for the establishment of the plant material. After 4 weeks, the number of nodes per shoot and number of shoots per explant were recorded and the MF was calculated as described previously.

The photosynthetic pigments were extracted from $100 \mathrm{mg}$ of fresh leaves. The extraction was performed in $15-\mathrm{mL}$ test tubes with $4 \mathrm{~mL}$ of methanol $100 \%$, heated $10 \mathrm{~min}$ in a water bath to $65^{\circ} \mathrm{C}$, and then stored at $4{ }^{\circ} \mathrm{C}$ for $24 \mathrm{~h}$. After centrifugation at $5000 \mathrm{~g}$ for $5 \mathrm{~min}$, the concentrations of total chlorophyll, chlorophyll $\mathrm{a}$ and $\mathrm{b}$ contents were determined spectrophotometrically (Lichtenthaler, 1987) with a spectrophotometer EVO 60 (Thermo Fisher Scientific Inc., Waltham, MA). Furthermore, the chlorophyll $a /$ chlorophyll $b$ ratio was calculated. Five samples for each treatment were analyzed and repeated three times. The dry matter was determined using 10 leaves for each treatment by heating at $105 \pm 2{ }^{\circ} \mathrm{C}$ and the ratio between dry weight and fresh weight were considered.

In vitro, ex vitro rooting, and acclimatization. At the end of multiplication phase, apical shoots with three to four nodes $(15-20 \mathrm{~mm}$ ) that developed on DKW medium supplemented with sucrose $3 \% \mathrm{w} / \mathrm{v}$ and BAP 0.5 $\mathrm{mg} / \mathrm{L}$ were excised and used for rooting experiments. The rooting media consisted of half-strength MS medium, supplemented with sucrose $1 \% \mathrm{w} / \mathrm{v}$, and two concentrations of IBA $(0.5$ and $1 \mathrm{mg} / \mathrm{L})$ and a hormone-free medium (as control) with or without putrescine $(160 \mathrm{mg} / \mathrm{L})$. Putrescine and IBA were added filter-sterilized after the sterilization.

The $\mathrm{pH}$ of the media was adjusted to 5.8 with $\mathrm{KOH} 1 \mathrm{~m}$ before adding $0.58 \%(\mathrm{w} / \mathrm{v})$ of plant agar (Duchefa Biochemie, B.V.). The cultures were kept at $24 \pm 1{ }^{\circ} \mathrm{C}$ in dark conditions for a week and then transferred under a 16 -h photoperiod with $40 \mu \mathrm{mol} \cdot \mathrm{m}^{-2} \cdot \mathrm{s}^{-1}$ light intensity provided by cool-white fluorescent lamps.
After 3 weeks of culture on rooting media, the percentage of rooted plantlets was calculated, and after another 2 weeks, explant survival was recorded. With the aim of shortening the micropropagation phase, ex vitro rooting experiments were performed, combining the rooting and acclimatization stages. In this case, the shoots were excised and dipped for $30 \mathrm{~s}$ in IBA $100 \mathrm{mg} / \mathrm{L}$, compared with the control (shoot dipped in distilled water), and placed in a Jiffy pots and covered with polyethylene film to maintain high relative humidity. After 3 weeks, the number of whole plantlets were recorded.

Data collection and statistical analysis. All experiments were repeated three times, and the data were used to perform statistical analysis. Analysis of variance was performed, and the means were separated by Tukey's test $(P<0.01)$. Data are presented as mean \pm SD.

\section{Results and Discussion}

Growth regulators and mineral basal salts used. The sterilization procedure used was very efficient for in vitro culture establishment, with $\approx 90 \%$ of aseptic explants. After just $10 \mathrm{~d}$, the explants developed at least one shoot suitable for the successive subcultures. Nodal segments are a preferred explant for in vitro propagation due to the presence of pre-existing meristems, which can develop into shoots maintaining clonal fidelity (Frabetti et al., 2009).

As previously described, a first experiment was performed, and different concentrations of BAP, ZR, and 2-iP were used to induce shoot proliferation. Figure 1 shows the effects of increasing concentrations $(0.1$, $0.5,1$, and 2) of BAP (Fig. 1A-D), 2-iP (Fig. 1E-F) and ZR (Fig. 1I-L) on the average number of shoots per explants, the average nodes per shoots, and MF after 4 weeks in culture, using a hormone-free medium as control.

As reported in Table 1, the use of BAP at different concentrations produced the best results among the treatments used; the concentrations ranging from 0.1 and $2 \mathrm{mg} / \mathrm{L}$ showed values ranging between 2.58 and 8.58 , higher than the other media. ZR and 2-iP were not able to induce multiple shoot formation and, in both cases, the number of shoots per explants was lower than the control (hormone-free medium).

Similarly, the number of nodes per shoots was influenced by the cytokinins used. 2-iP and ZR negatively affected explant growth, since the average values were lower than the control (hormone-free). The presence of BAP promoted new node organogenesis, particularly at 0.5 and $1 \mathrm{mg} / \mathrm{L}$, whereas the highest concentration $(2 \mathrm{mg} / \mathrm{L})$ induced less shoot development. The MF showed that proliferation was highest in medium containing BAP at concentrations of $0.5,1$, and $2 \mathrm{mg} / \mathrm{L}(20.93$, 65.35 , and 46.53 , respectively).

Abundant basal callus formation was stimulated in 2-iP-enriched media at all concentrations, unlike the explants grown on ZR-enriched media, in which neither growth nor callus formation was observed. In addition, the use of 1 and $2 \mathrm{mg} / \mathrm{L}$ of BAP stimulated the callus growth at the base of explants.

On the basis of these results obtained, we opted to proceed with experiments comparing basal media using BAP at $0.5 \mathrm{mg} / \mathrm{L}$, first because at this concentration the explants did not induce callus formation and second because this concentration provides welldeveloped explants with lower MF but with longer and easily-to-manipulate explants.

Results from this study agree with previous reports that BAP was the effective cytokinin for in vitro shoot multiplication of wolfberry (Fira et al., 2016), and the changes in the type and concentration of cytokinin applied can drastically affect shoot proliferation. The presence of callus formation and hyperhydricity at higher concentrations of BAP has been observed; these findings are confirmed in other species, where a dosedependent manner of cytokinins involved in hyperhydricity has been observed (Liu et al., 2017).

Four weeks of growth in MS, DKW, QL, and WPM showed the significant influence of media mineral composition (Fig. 2) on the studied parameters (Table 2). On MS and DKW media, maximum shoot multiplication was recorded, in comparison with QL and WPM. According to the results, mean shoots per explant were highest on DKW medium (6.73), followed by MS (4.30), compared with QL and WPM media, that negatively affected by the medium with an average of 1.85 and 1.11 shoots per explant, respectively. The average number of nodes per shoot was characterized by mean values ranging between 6.38 on MS medium and 7.88 in WPM medium.

The MF showed that overall proliferation rate was highest on DKW medium. In particular, QL and WPM media resulted in very low proliferation, confirming that these basal media are not suitable for wolfberry micropropagation.

The analysis of chlorophyll content revealed that on MS medium the shoots have a significantly higher pigment content than QL and WPM media, whereas the ratio between chlorophyll a and chlorophyll b was not different among the media used, except for WPM, where this ratio was lower than the other media (Table 2).

The optimization of in vitro culture conditions through the study of basal salts and growth regulators has an important impact on the rooting and acclimatization processes. Previous reports described the use of MS medium (Fira et al., 2016), but in our experiments, the use of these basal salts, although achieving good results in terms of plant growth, during the second and third subculture, the shoots showed detrimental effects, characterized by a drastic growth reduction and hyperhydricity (data not shown). Our results suggest that the DKW medium is the most appropriate medium 


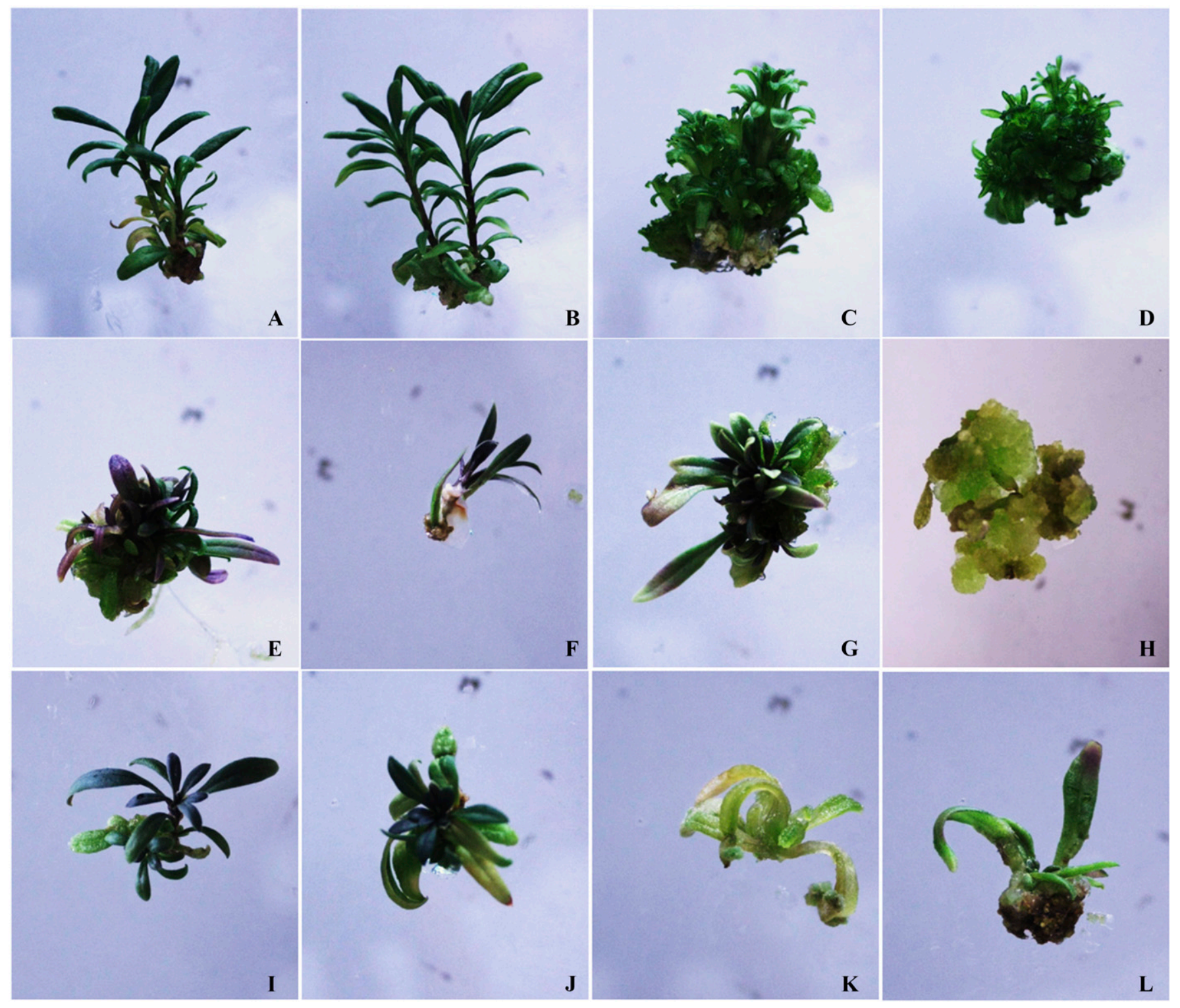

Fig. 1. In vitro shoot culture of wolfberry cv. Nixia 1 . Shown are $0.1,0.5,1$, and $2 \mathrm{mg} / \mathrm{L}$ of (A-D) 6-benzylaminopurine (BAP), (E-H) N6-(2-Isopentenyl)adenine (2-iP), and (I-L) zeatin (ZR).

Table 1. Effect of MS basal medium supplemented with different PGRs on induction and development of axillary shoots of wolfberry, cv. Nixia $1 .{ }^{\mathrm{z}}$

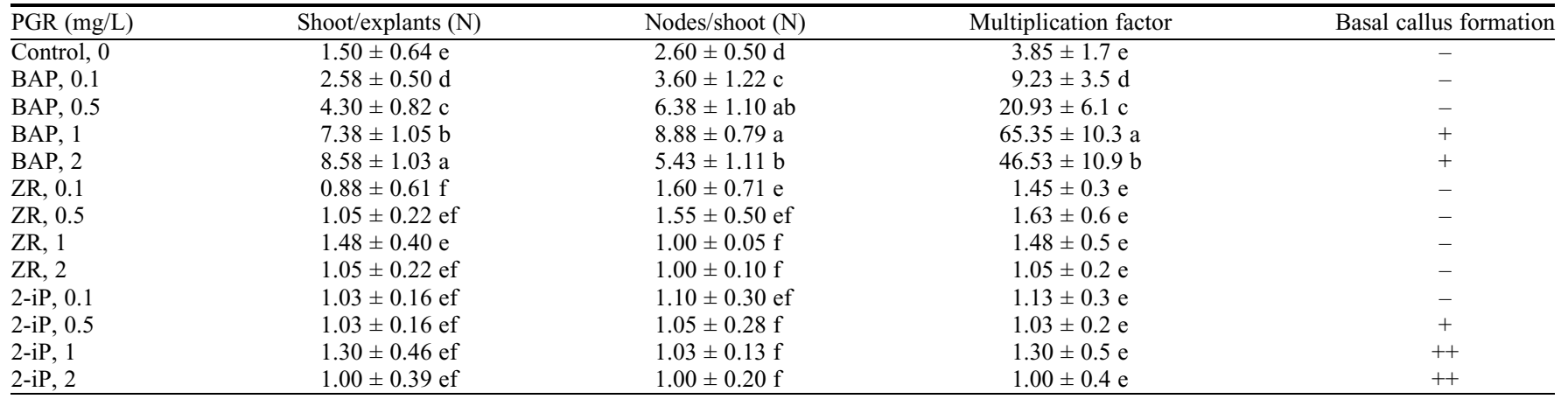

${ }^{\mathrm{z}}$ Data are presented as mean \pm SD. Different letters within the same columns indicated significant differences among the treatments (Tukey's test, $P<0.01$ ). $\mathrm{MS}=$ Murashige and Skoog; PGR = plant growth regulator; BAP = 6-benzylaminopurine; ZR = zeatin; 2-iP = N6-(2-isopentenyl)adenine.

among the media tested; its effectiveness in shoot induction and development could be related to the mineral composition and their concentrations and, as for many woody species, Lycium barbarum prefers media with low ion concentrations. On the contrary, shoot growth on the MS medium tended toward hyperhydricity.

The effectiveness of DKW medium in shoot growth and development could be re- lated to the medium mineral compositions and their concentrations. The mineral concentrations of the MS medium are too high for many woody species and increasing the number of subcultures provokes an increase 
Table 2. Comparison among different basal media on several parameters of growth of wolfberry, cv. Nixia 1, with BAP 0.5 mg/L. ${ }^{z}$

\begin{tabular}{|c|c|c|c|c|c|c|}
\hline Basal salts (including vitamins) & Shoot/explants (N) & Nodes/shoot $(\mathrm{N})$ & Multiplication factor & Chl total $(\mathrm{mg} / \mathrm{g} \mathrm{FW})$ & $\mathrm{Chl} \mathrm{a/Chl} \mathrm{b}$ & DW/FW ratio \\
\hline WPM & $1.11 \pm 0.22 \mathrm{c}$ & $7.88 \pm 0.95 \mathrm{a}$ & $8.75 \pm 1.4 \mathrm{c}$ & $1.04 \pm 0.04 \mathrm{~b}$ & $2.010 \pm 0.09 \mathrm{~b}$ & $0.097 \pm 0.08 \mathrm{c}$ \\
\hline DKW & $6.73 \pm 0.40 \mathrm{a}$ & $7.45 \pm 0.43 a b$ & $38.08 \pm 8.1 \mathrm{a}$ & $1.52 \pm 0.04 \mathrm{ab}$ & $2.278 \pm 0.06 \mathrm{a}$ & $0.130 \pm 0.03 \mathrm{a}$ \\
\hline
\end{tabular}

${ }^{\mathrm{z}}$ Data are presented as mean $\pm \mathrm{SD}$. Different letters within the same columns indicated significant differences among the treatments (Tukey's test, $P<0.01$ ). $\mathrm{BAP}=6$-benzylaminopurine; $\mathrm{Chl}=$ chlorophyll; $\mathrm{FW}=$ fresh weight; $\mathrm{Chl} \mathrm{a}=$ chlorophyll $\mathrm{a} ; \mathrm{Chl} \mathrm{b}=$ chlorophyll b; DW = dry weight; MS = Murashige and Skoog; $\mathrm{WPM}=$ Woody Plant Medium; DKW = Driver and Kuniyuki Walnut.
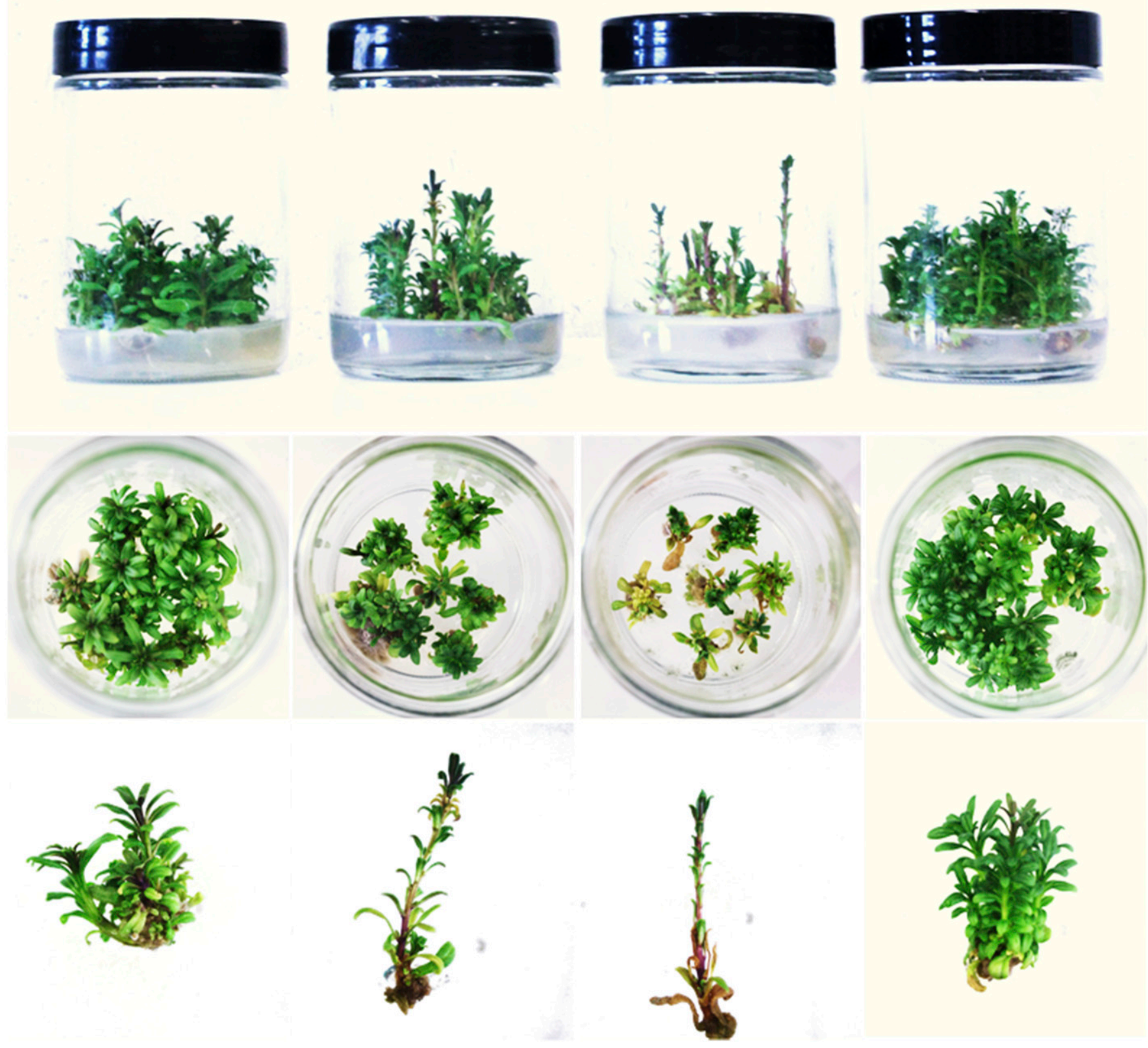

Fig. 2. In vitro shoot culture of wolfberry cv. Nixia 1 on media Murashige and Skoog (MS), Quoirin and Lepoivre (QL), Woody Plant Medium (WPM), and Driver and Kuniyuki Walnut (DKW) in order from left to right.

of hyperhydricity, probably due to the ratio of $\mathrm{NH}_{4}^{+}$and $\mathrm{NO}_{3}^{-}$of MS medium (Ivanova and Van Staden, 2009), a medium with high $\mathrm{NH}_{4}^{+}$, which represents a great growth-limiting stress to plants (Sarasketa et al., 2016).

In vitro, ex vitro rooting, and acclimatization. As reported in Table 3, the percentage of rooted shoots was very high in half-strength MS medium containing sucrose $1 \%$ and both concentrations of IBA with or without putrescine. The percentage of acclimatization of plants in soil ranging between $88.7 \%$ and $95.1 \%$. No significant differences were observed among the treatments used.

Many authors have demonstrated that rooting takes place because of a series of physiological phases that are associated with changes in peroxidase activity and endogenous auxin concentrations, and often polyamine concentrations are used as markers of the rooting process (Rugini, 1992). Putrescine, alone or combined with auxin, usually advances the rooting process, increasing the rooting percentage and the number of 
Table 3. In vitro and ex vitro rooting and shoot acclimatization percentage of L. barbarum L. ${ }^{\mathrm{z}}$

\begin{tabular}{lcc}
\hline Rooting media & Rooting (\%) & Acclimation (\%) \\
\hline IBA (0.5) & $87.2 \pm 5.5$ & $90.0 \pm 6.2$ \\
IBA (0.5) + Put (160) & $94.2 \pm 3.4$ & $95.1 \pm 7.4$ \\
IBA (1) & $89.2 \pm 5.2$ & $88.7 \pm 6.8$ \\
IBA (1) + Put (160) & $91.6 \pm 6.1$ & $92.2 \pm 7.4$ \\
Dipping in IBA 100 mg/L (ex vitro) & $95.2 \pm 7.2$ & - \\
\hline
\end{tabular}

${ }^{\mathrm{z}}$ Data are presented as mean $\pm \mathrm{SD}$

$\mathrm{IBA}=$ indole-3-butyric acid; Put $=$ putrescine.

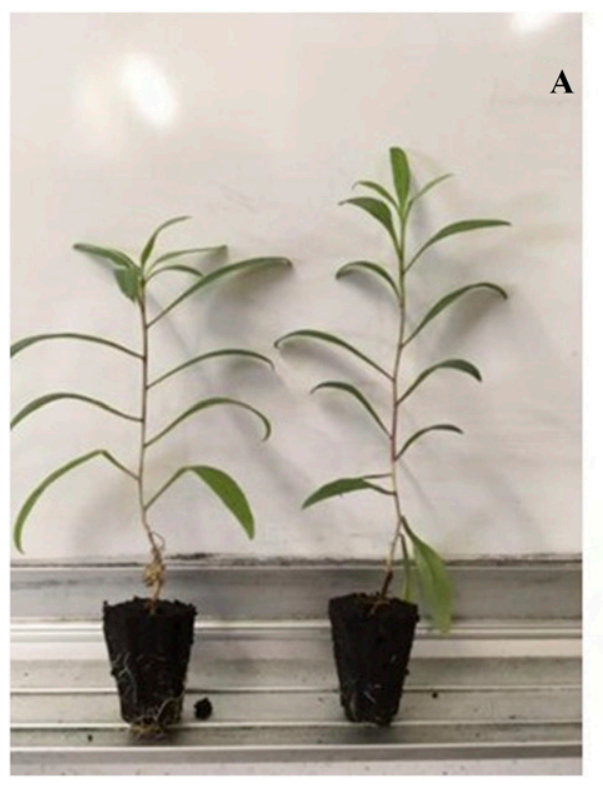

Fig. 3. (A) Plantlets of wolfberry rooted ex vitro. (B) Well-developed adventitious rootlets after 3 weeks of acclimatization.

neoformed roots (Mendoza de Gyves et al., 2007). In our experiments, the application of exogenous putrescine showed positive effects on rooting when combined with a low concentration of auxin, as already observed in previous works (Geneve and Kester, 1991).

The ex vitro rooting of wolfberry dipped in IBA showed a very good rooting percentage (Table 3) not different from the experiments of in vitro rooting, and the plantlets acclimatized well in ex vitro conditions (Fig. 3), demonstrating for this species that ex vitro rooting can be a way to shorten the propagation protocol.

\section{Conclusions}

This work describes a systematic approach for developing an in vitro micropropagation protocol for wolfberry, showing the highest rate of proliferation reported for this species compared with existing literature. The protocol differs from the previously reported methods, because the explants used were derived from a commercial cultivar and not from seedlings. Our results demonstrated that the use of DKW can benefit in vitro culture because these basal salts, contrary to MS, did not induce vitrification of the explants. Since the interest in wolfberry cultivation in recent years has steadily increased, nursery industries have the need to produce genetically uniform 31:195-201.
B

Driver, J.A. and A.H. Kuniyuki. 1984. In vitro propagation of Paradox walnut rootstock. HortScience 19:507-509.

Fira, A., N. Joshee, V. Cristea, M. Simu, M. Harta, D. Pamfil, and D. Clapa. 2016. Optimization of micropropagation protocol for Goji berry (Lycium barbarum L.). Bull. Univ. Agr. Sci. Veterinary Med. Cluj-Napoca. Hort. 73:141150.

Frabetti, M., P. Gutiérrez-Pesce, E. Mendoza-De Gyves, and E. Rugini. 2009. Micropropagation of Teucrium fruticans L., an ornamental and medicinal plant. In Vitro Cell. Dev. Biol. Plant 45:129-134.

Fukuda, T., J. Yokoyama, and H. Ohashi. 2001. Phylogeny and biogeography of the genus Lycium (Solanaceae): Inferences from chloroplast DNA sequences. Mol. Phylogenet. Evol. 19(2):246-258.

Geneve, R.L. and S.T. Kester. 1991. Polyamines and adventitious root formation in the juvenile and mature phase of English ivy. J. Exp. Bot. 42:71-75.

Hu, Z., G.Q. Guo, D.L. Zhao, L.H. Li, and G.C. Zheng. 2001. Shoot regeneration from cultured leaf explants of Lycium barbarum and Agrobacterium-mediated transformation. Russ. J. Plant Physiol. 48:529-535.

$\mathrm{Hu}, \mathrm{Z}$., Y. Hu, H.H. Gao, and G.C. Zheng. 2002. High-efficiency transformation of Lycium barbarum mediated by Agrobacterium tumefaciens and transgenic plant regeneration via somatic embryogenesis. Plant Cell Rep. 21:233-237.

Hu, Z., Y.R. Wu, W. Li, and H.H. Gao. 2006. Factors affecting Agrobacterium tumefaciens mediated genetic transformation of Lycium barbarum L. In Vitro Cell. Dev. Biol. Plant 42:461-466.

Ivanova, M. and J. Van Staden. 2009. Nitrogen source, concentration, and $\mathrm{NH}_{4}(+)$ : $\mathrm{NO}_{3}(-)$ ratio influence shoot regeneration and hyperhydricity in tissue cultured Aloe polyphylla. Plant Cell Tissue Organ 99:167-174.

material from selected genotypes, and the presence of reliable and efficient protocols is important for economic production. The use of putrescine in vitro was beneficial to rooting, as reported in the literature for several species, but only when combined with low concentration of auxin, by strengthening its activity, which could be very helpful in those explants whose buds are inhibited by high doses of auxin, negatively influencing the budbreaking. However, we show that ex vitro rooting is very effective, improving not only rooting but also plantlet acclimatization. This procedure, combined with high proliferation medium and the high quality of shoots recovered, makes this protocol very effective and could support rapid growth in the commercial propagation of wolfberry varieties.

\section{Literature Cited}

Benmahioul, B., N. Dorion, M. Kaid-Harche, and F. Daguin. 2012. Micropropagation and ex vitro rooting of pistachio (Pistacia vera L.). Plant Cell Tissue Organ Cult. 108:353358.

Cui, K.R., X.W. Pei, J.J. Wang, and Y.F. Wang. 1998. Effects of modulation of abscisic acid during somatic embryogenesis in Lycium barbarum L. Acta Biologiae Experimentalis Sinica

Cui, K.R., G.S. Xing, X.M. Liu, G.M. Xing, and Y.F. Wang. 1999. Effect of hydrogen peroxide on somatic embryogenesis of Lycium barbarum L. Plant Sci. 146:9-16.
Lichtenthaler, H. 1987. Chlorophylls and carotenoids: Pigments of photosynthetic membranes. Methods Enzymol. 148:350-382.

Liu, M., F. Jiang, X. Kong, J. Tian, Z. Wu, and Z. Wu. 2017. Effects of multiple factors on hyperhydricity of Allium sativum L. Scientia Hort. 217:285-296.

Lloyd, G. and B. McCown. 1980. Commerciallyfeasible micropropagation of mountain laurel, Kalmia latifolia, by use of shoot tip culture. Intl. Plant Prop. Soc. Proc. 30:421-427.

Mendoza de Gyves, E., J. I. Royani, and E. Rugini. 2007. Efficient method of micropropagation and in vitro rooting of teak (Tectona grandis L.) focusing on large-scale industrial plantations. Ann. For. Sci. 64(1):73-78.

Murashige, T. and F. Skoog. 1962. A revised medium for rapid growth and bioassays with tobacco tissue cultures. Physiol. Plant. 15:473-479.

Nalawade, S.M., A.P. Sagare, C.Y. Lee, C.L. Kao, and H.S. Tsay. 2003. Studies on tissue culture of Chinese medicinal plant resources in Taiwan and their sustainable utilization. Bot. Bull. Acad. Sin. 44:79-98.

Okemo, P.O., H.P. Bais, and J.M. Vivanco. 2003. In vitro activities of Maesa lanceolata extracts against fungal plant pathogens. Fitoterapia 74:312-316.

Osman, N.I., A. Awal, N.J. Sidik, and S. Abdullah. 2012. Antioxidant activities of in vitro seedlings of Lycium barbarum (Goji) by diphenyl picrylhydrazyl (DPPH) assay. Int. J. Pharm. Pharm. Sci. 4(Suppl.):137-141.

Osman, N.I., A. Awal, N.J. Sidik, and S. Abdullah. 2013a. Callus induction and somatic embryogenesis 
from leaf and nodal explants of Lycium barbarum L. (Goji). Biotechnology (Faisalabad) 12(1):3645 .

Osman, N.I., A. Awal, N.J. Sidik, and S. Abdullah. 2013 b. In vitro regeneration and antioxidant properties of Lycium barbarum L. (goji). J. Teknologi 62:35-38.

Osuna, L., M.E. Tapia-Pérez, O. Figueroa, E. Jiménez-Ferrer, M.L. Garduño-Ramírez, M.T. González-Garza, P. Carranza Rosales, and D.E. Cruz-Vega. 2006. Micropropagation of Lepidium virginicum (Brassicaceae), a plant with antiprotozoal activity. In Vitro Cell. Dev. Biol. Plant 42:596-600.

Parveen, V., V.K. Sharma, and A.K. Mandal. 2005. A protocol for micropropagation of an important medicinal plant-Oroxylum indicum Vent. through cotyledonary nodal explants. Ann. For. (Dehra Dun) 13:53-58.
Potterat, O. 2010. Goji (Lycium barbarum and $L$. chinense): Phytochemistry, pharmacology and safety in the perspective of traditional uses and recent popularity. Planta Med. 76(1):7-19.

Quoirin, M. and P. Lepoivre. 1977. Improved media for in vitro culture of Prunus sp. Acta Hort. 78:437-442.

Rugini, E. 1992. Involvement of polyamines in auxin and Agrobacterium rhizogenes-iduced rooting of fruit trees in vitro. J. Amer. Soc. Hort. Sci. 117:532-553.

Saiju, H.K. 2005. Tree tissue culture and ex vitro sand rooting for reforestation, p. 151-154. In: K. Suzuki, K. Ishii, S. Sakurai, and S. Sasaki (eds.). Plantation technology in tropical forest science. Springer, Berlin.

Sarasketa, A., M.B. Gonzalez-Moro, C. GonzalezMurua, and D. Marino. 2016. Nitrogen source and external medium $\mathrm{pH}$ interaction differentially affects root and shoot metabolism in Arabidopsis. Front. Plant Sci. 7:29.

Seeram, N.P. 2008. Berry fruits: Compositional elements, biochemical activities, and the impact of their intake on human health, performance, and disease. J. Agr. Food Chem. 56(3):627-629.

Sze, S.C.W., J.X. Song, R.N.S. Wong, Y.B. Feng, T.B. Ng, Y. Tong, and K.Y.B. Zhang. 2008. Application of SCAR (sequence characterized amplified region) analysis to authenticate Lycium barbarum (wolfberry) and its adulterants. Biotechnol. Appl. Biochem. 5:15-21.

Zhang, K.Y.B., H.W. Leung, H.W. Yeung, and R.N.S. Wong. 2001. Differentiation of Lycium barbarum from its related Lycium species using random amplified polymorphic DNA. Planta Med. 67:379-381. 